\title{
Hepatitis autoinmune asociada al uso de adalimumab en un paciente con artritis reumatoide
}

\author{
Autoimmune hepatitis associated \\ to adalimumab in a patient with rheumatoid \\ arthritis
}

\author{
Natalia Aristizábal, Carlos Jaime Velásquez, Luis Fernando Pinto • \\ Medellín (Colombia)
}

\begin{abstract}
Resumen
La hepatitis autoinmune es una entidad progresiva de causa desconocida. En su patogénesis, se han propuesto disparadores ambientales, como virus y drogas, que al actuar sobre un paciente predispuesto genéticamente, desencadenan eventos mediados por células T, llevando a injuria hepatocelular. Los antagonistas del factor de necrosis tumoral son medicamentos utilizados para el manejo de la artritis reumatoide. Se ha demostrado, sin embargo, que pueden generar daño hepático por diferentes mecanismos, uno de ellos la inducción de hepatitis autoinmune; la mayoría de los casos publicados se han asociado al uso de infliximab o etanercept. Se presenta un caso de hepatitis autoinmune inducido por adalimumab en una paciente con artritis reumatoide. (Acta Med Colomb 2012; 37: 147-151)

Palabras clave: hepatitis autoimmune, adalimumab, artritis reumatoide, hipergammaglobulinemia, autoanticuerpos.
\end{abstract}

\begin{abstract}
Autoimmune hepatitis is a progressive autoimmune disease of unknown cause. In regard to its pathogenesis, environmental triggers such as viruses and drugs have been proposed . These triggers when acting on a genetically predisposed patient, trigger T cell-mediated events, leading to hepatocellular injury. Tumor necrosis factor antagonists are drugs used for the management of rheumatoid arthritis. It has been shown, however, that they can generate liver damage by different mechanisms, one of which is induction of autoimmune hepatitis. Most of the reported cases have been associated with use of infliximab or etanercept. We report a case of autoimmune hepatitis induced by adalimumab in a patient with rheumatoid arthritis. (Acta Med Colomb 2012; 37: 147-151)

Keywords: autoimmune hepatitis, adalimumab,rheumatoid arthritis, hypergammaglobulinemia, antibodies.
\end{abstract}

Dra. Natalia Aristizábal Henao: Residente Tercer Año de Medicina Interna, Universidad Pontificia Bolivariana; Dres. Carlos Jaime Velásquez Franco y Luis Fernando Pinto Peñaranda: Internistas Reumatólogos Hospital Pablo Tobón Uribe. Profesores de Medicina Interna, Facultad de Medicina. Escuela de Ciencias de la Salud, Universidad Pontificia Bolivariana. Medellín (Colombia).

Correspondencia. Dr. Carlos Jaime Velásquez Franco. Medellín (Colombia).

E-mail: carjaivel@hotmail.com.

Recibido: 15/XII/2011 Aceptado: 20/VIII/2012

\section{Introducción}

La hepatitis autoinmune es una entidad generalmente progresiva de causa desconocida, que ocurre en personas de todas las edades (1). Se caracteriza por inflamación hepática crónica, hipergamaglobulinemia y producción de autoanticuerpos (2).

Nuevos estudios clínicos y de ciencias básicas han mostrado hallazgos importantes que contribuyen al entendimiento de la patogénesis y las alternativas terapéuticas en esta entidad (2). Actualmente se postula que existen agentes ambientales capaces de disparar una cascada de eventos mediados por células $\mathrm{T}$ dirigidos contra antígenos hepáticos en personas con predisposición genética, causando inflamación, necrosis y fibrosis (1). Los agentes ambientales que inducen hepatitis autoinmune no están completamente definidos, pero se sabe que incluyen virus y algunos medicamentos están implicados.

La artritis reumatoide es la artropatía inflamatoria más común, con una prevalencia que oscila entre el $0.5-1 \%$ de la población general. En la actualidad existen herramientas 
diagnósticas que permiten su detección precoz y manejo agresivo cuyo objetivo es inducir remisión. Actualmente se cuentan con varios medicamentos que permiten modular la respuesta biológica exagerada que se presenta en esta entidad; entre ellos, están los antagonistas del factor de necrosis tumoral (anti-TNF) (1). Varios casos de hepatitis autoinmune inducidos por estos fármacos han sido reportados, la mayoría asociados a infliximab (anticuerpo monoclonal quimérico) $\mathrm{y}$, en menor medida, a etanercept (proteína de fusión).

Se presenta un caso de hepatitis autoinmune en una paciente con artritis reumatoide tratada con adalimumab (anticuerpo monoclonal humanizado anti-TNF).

\section{Presentación de caso}

Mujer de 28 años, sin antecedentes patológicos, alérgicos, tóxicos ni quirúrgicos de importancia, con historia clínica de artralgias generalizadas desde los 20 años de edad, cada vez de mayor intensidad, evolucionando a poliartritis recurrente establecida desde los 23 años, con las características palindrómicas; a pesar del uso de cloroquina, la paciente presenta poliartritis sumatoria persistente y progresiva, sin otros síntomas o signos asociados, por lo cual se adiciona al manejo metotrexate, sin lograr resolución completa de la inflamación articular a pesar de recibir altas dosis (hasta $20 \mathrm{mg}$ semanales). Se prescribió además sulfasalazina por 12 semanas ( 2 gr diarios), sin respuesta. En la revisión por sistemas hay ausencia de síntomas secos y ausencia de compromiso órgano específico. El perfil de autoinmunidad de la paciente se presenta en la Tabla 1.

Dos años más tarde se hace diagnóstico clínico de artritis reumatoide, por poliartritis florida que comprometía manos y carpos y rigidez matinal de más de una hora de duración. Se inicia manejo con leflunomida a dosis plenas $(20 \mathrm{mg}) \mathrm{sin}$ lograr respuesta clínica. Cuatro meses después se decide iniciar adalimumab $40 \mathrm{mg}$ subcutáneos (SC) cada dos semanas. Posterior al inicio del agente biológico (aproximadamente a las ocho semanas), la paciente presenta remisión completa de su enfermedad desde el punto de vista clínico y de laboratorio, con adecuada tolerancia al medicamento. Debido a

Tabla 1. Perfil de autoinmunidad.

\begin{tabular}{|ll|}
\hline Año & \multicolumn{1}{c|}{ Perfil de autoinmunidad } \\
\hline 2002 & $\begin{array}{l}\text { Anticuerpos antinucleares (ANA) con dilución 1:1280 patrón anticito- } \\
\text { plasmático (tipo aparato de Golgi). }\end{array}$ \\
\hline 2003 & $\begin{array}{l}\text { AntiDNA de doble cadena (ds) y anti-ENA (antígenos extractables del } \\
\text { núcleo) negativos. } \\
\text { ANA 1:1280 patrón anticitoplasmático (tipo aparato de Golgi). }\end{array}$ \\
\hline 2004 & ANA 1:640 patrón anticitoplasmático (tipo aparato de Golgi). \\
\hline 2005 & $\begin{array}{l}\text { Anti-DNA ds y anti-ENAs negativos. } \\
\text { ANA 1:640 patrón anticitoplasmático (tipo aparato de Golgi). }\end{array}$ \\
\hline 2007 & $\begin{array}{l}\text { Anti-ENA, antiDNA ds, ANA, anticuerpos anticitrulina ( anti-CCP) y } \\
\text { factor reumatoide (FR) negativos. }\end{array}$ \\
\hline
\end{tabular}

la evolución clínica favorable, se suspende el antimalárico y se inicia el desmonte de metotrexate.

Dos años después del inicio del adalimumab, es detectado un aumento progresivo de las aminotransferasas, con una mayor elevación de la alanino aminotransferasa (ALT), por lo que se suspende definitivamente el metotrexate (Figura 1).

Los estudios para virus de la hepatitis A, B o C, y citomegalovirus (CMV) fueron negativos; los valores de la bilirrubina total y la gamaglutamiltranspeptidasa se encontraban en rangos de normalidad; la ecografía hepática no demostró alteraciones en el parénquima hepático ni lesiones asociadas.

Las transaminasas persistían elevadas e incrementándose pese a la suspensión de adalimumab y de algunos productos herbales que consumía la paciente.

Se decide entonces realizar biopsia hepática, en la cual se evidenció inflamación crónica activa con preservación de la arquitectura lobulillar, tumefacción generalizada de hepatocitos, infiltrado mononuclear portal y en interfase, apolillamiento y necrosis moderada de hepatocitos aisla-

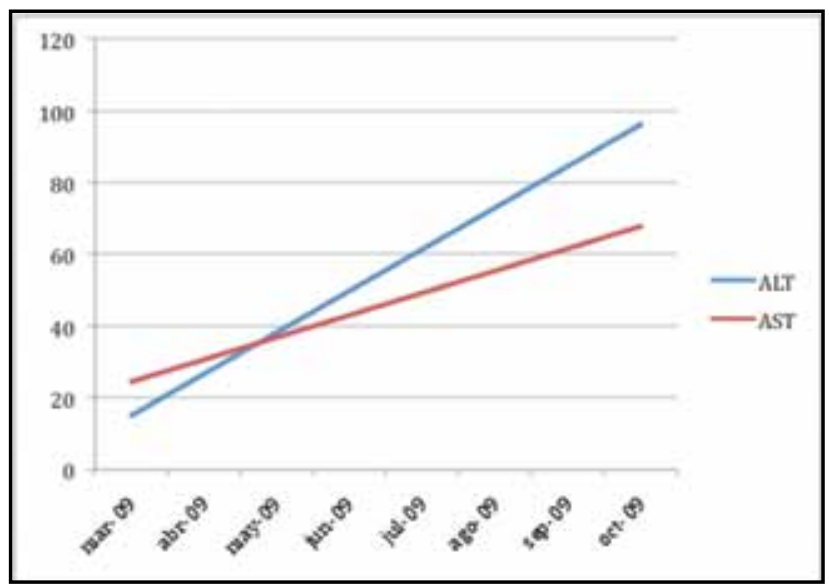

Figura 1. Comportamiento de las aminotransferasas dos años después del inicio de adalimumab $(U I / L)$.

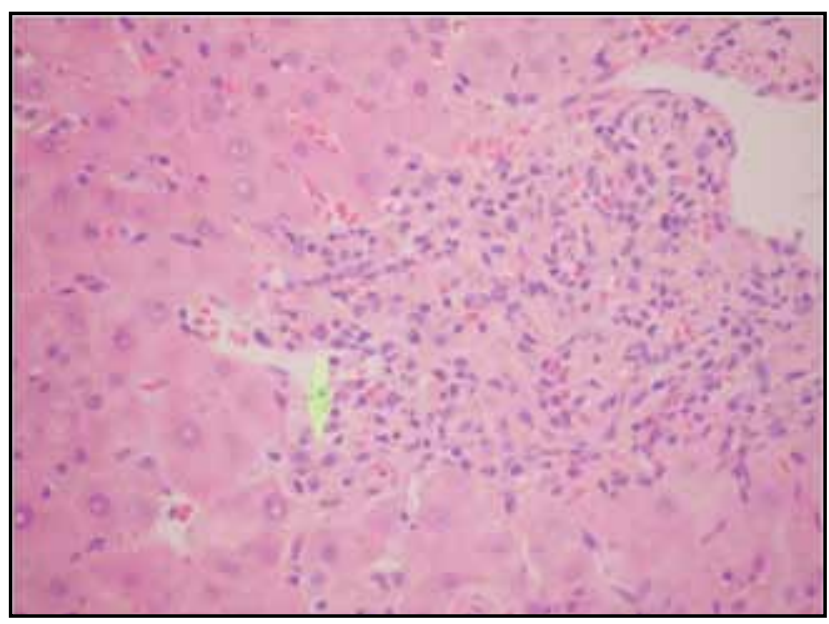

Figura 2. Estudio anatomopatológico obtenido de biopsia hepática con evidencia de espacio porta con moderado infiltrado inflamatorio mononuclear con predominio de plasmocitos. Leve actividad de interfase. La fecha muestra acúmulo de plasmocitos portales. Coloración de hematoxilina-eosina a un aumento de $10 X$. 
dos y se observó una porción lobular con múltiples focos de infiltrado linfoplasmocitario, compatible con hepatitis autoinmune. No se encontró esteatosis, ni hepatocitos en vidrio esmerilado (Figuras 2 y 3 ).

La paciente se manejó con azatioprina $75 \mathrm{mg} /$ día y prednisolona $30 \mathrm{mg}$ diarios, presentando una evolución satisfactoria, con normalización completa del perfil hepático a los tres meses (Figura 4).

\section{Discusión}

El uso de medicamentos anti TNF está plenamente validado en ciertas condiciones autoinmunes como la artritis reumatoide, donde alcanza altas tasas de respuesta. Sin embargo, se ha encontrado que estas drogas pueden causar injuria hepatocelular mediada por diferentes mecanismos, tales como la reactivación de hepatitis B en portadores, inducción de hepatitis autoinmune, hepatitis tóxica y producción de falla hepática aguda en ciertos pacientes (3). Se ha descrito que pueden inducir la formación de autoanticuerpos, por un mecanismo desconocido, aunque se han postulado teorías en relación con la pérdida de supresión de la respuesta inmune en presencia del medicamento (4);

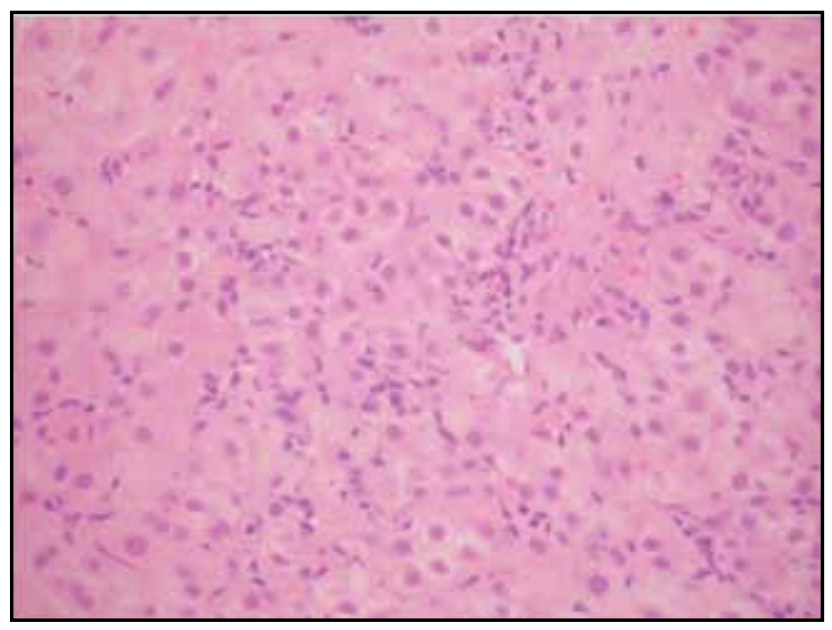

Figura 3. Parénquima hepático con actividad lobulillar moderada, pseudorrosetas, hepatocitos necróticos e hiperplasia de células de Kupffer. Coloración de hematoxilina eosina. Aumento de 40X.

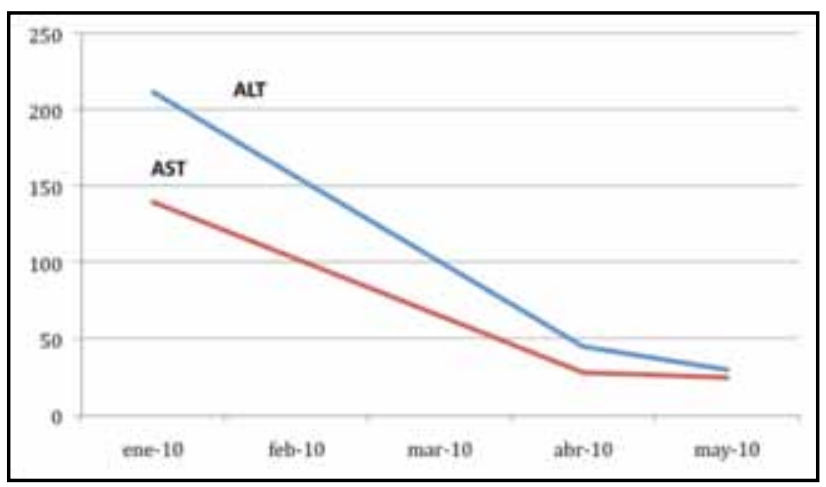

Figura 4. Valores de transaminasas (UI/L) al iniciar manejo con azatioprina y prednisolona. además, se han encontrado anticuerpos dirigidos contra los propios agentes anti-TNF, antihumanos (contra adalimumab y etanercept) y antiquiméricos (antiinfliximab) (5).

La hepatitis autoinmune es una entidad inflamatoria crónica, que ocurre especialmente en mujeres jóvenes y en pacientes con otras enfermedades autoinmunes (1); se caracteriza ocasionalmente por tener un curso fluctuante con periodos de recaída y remisión (1). Es el resultado del desarrollo de una respuesta inmune contra antígenos propios o externos, con porciones moleculares similares (mimetismo molecular), que sobrepasa los mecanismos reguladores y supresores fisiológicos (2) en pacientes con predisposición genética expuestos a ciertos agentes ambientales; estos disparadores no están completamente identificados, pero a la fecha se reconoce el papel de ciertos virus y algunos medicamentos, como los agentes anti $\mathrm{TNF}$, en la inducción de estas respuestas anómalas (1). Una posible susceptibilidad para la hepatitis autoinmune relacionada con genes del TNF está en estudio, pero los resultados son contradictorios (1).

Ciertos medicamentos pueden inducir injuria hepatocelular simulando una hepatitis autoinmune (1), además algunos productos herbales producen hepatotoxicidad e inducen respuestas inmunes anormales $(3,6)$. No es claro si las drogas y los productos herbales enmascaran o inducen una hepatitis autoinmune o simplemente causan una hepatitis tóxica que se acompaña de características autoinmunes (1).

El diagnóstico está basado en cambios histológicos, características clínicas, hallazgos bioquímicos y niveles aumentados de globulinas séricas incluyendo autoanticuerpos (1), parámetros que determinan sistemas de puntuación que indican gravedad y pronóstico (2).

El espectro clínico de la enfermedad es muy amplio; incluye pacientes completamente asintomáticos, hasta formas graves con ictericia y prolongación del tiempo de protrombina (TP) como marcadores de falla hepática aguda (1). La alteración predominante en el perfil bioquímico corresponde a elevación de las aminotransferasas, principalmente de la ALT. Sin embargo, algunos casos pueden mostrar un patrón colestásico con elevación de la bilirrubina total y fosfatasas alcalinas; casos en los cuales debe descartarse cirrosis biliar primaria asociada (1).

Los hallazgos en la biopsia son los de una hepatitis crónica, con algunos cambios característicos pero ninguno de ellos específico (1), como la presencia de un infiltrado de células mononucleares periportal que puede evolucionar a hepatitis lobular. Es frecuente la presencia de células plasmáticas y eosinófilos; conforme progresa la enfermedad se encuentra fibrosis en mayor o menor medida (1). La realización de biopsia hepática en los casos sospechosos de hepatitis autoinmune permite además descartar diagnósticos diferenciales. Muchos de los cambios histológicos descritos pueden revertir con la terapia instaurada (2).

Los pacientes con hepatitis autoinmune usualmente reciben terapia con esteroides y azatioprina, la cual es 
efectiva en $65-80 \%$ de los casos (1). Se ha descrito que hasta $9 \%$ de los pacientes progresa a pesar de la terapia y $50-86 \%$ recaen después de suspenderla (2). Algunos pacientes requieren terapia de mantenimiento con esteroides y/o azatioprina para evitar las recaídas frecuentes (1). El trasplante hepático se requiere en pacientes que son refractarios o intolerantes a la terapia inmunosupresora y desarrollan una hepatopatía en fase terminal (1).

Existen nuevas terapias propuestas para el manejo de la hepatitis autoinmune que pueden resultar efectivas en la artritis reumatoide. El uso de estas moléculas en hepatitis autoinmune, si bien promisorio, requiere la realización de estudios aleatorizados y controlados, que permitan tener evidencia suficiente para recomendarlas como terapias de primera línea (2).

Los agentes anti-TNF actualmente avaladas por la FDA (Food and Drug Administration) para uso en humanos son: infliximab, un anticuerpo monoclonal quimérico; etanercept, una proteína de fusión compuesta por receptores para el TNF; adalimumab, un anticuerpo monoclonal humanizado; certolizumab, un fragmento fijador del TNF conjugado con polietilenglicol; y golimumab, un anticuerpo humanizado subcutáneo (7).

En un estudio retrospectivo de 96 pacientes con artritis reumatoide y/o espondiloartritis que iniciaron manejo con etanercept o adalimumab como primer biológico, en el seguimiento a un año se encontró que once individuos suspendieron adalimumab por efectos secundarios, pero ninguno de ellos presentó eventos adversos hepáticos; se reportó un caso de lupus inducido por drogas. La toxicidad con adalimumab tuvo una relación directamente proporcional con el tiempo de uso (8).

Varios casos de hepatitis autoinmune inducidos por anti-TNF han sido reportados, la mayoría atribuidos a infliximab (9) y, en menor medida, a etanercept. Uno de los casos reportados con el uso de etanercept ocurrió en un paciente que había presentado hepatitis autoinmune previa inducida por infliximab (7).

En la literatura mundial se informa el caso de una paciente con diagnóstico de artritis reumatoide, quien desarrolló daño hepático severo después de nueve meses de tratamiento con leflunomida y adalimumab. Se evidencia en la biopsia cambios compatibles con hepatitis autoinmune y el cuadro se resuelve por completo con el uso de prednisolona y azatioprina. En este caso el uso de terapia combinada no permite establecer causalidad directa de ninguno de los dos medicamentos usados (10).

Sólo se encuentra en la literatura revisada un caso de hepatitis autoinmune inducida por adalimumab reportado en una mujer de 36 años con diagnóstico de artritis psoriásica, hipotiroidismo y enfermedad de Crohn con manifestaciones intestinales y extraintestinales. Esta paciente, luego de tres meses de tratamiento con adalimumab, presenta hepatitis autoinmune con título de ANA 1:80 (previo al tratamiento título de ANA negativo) y biopsia diagnóstica; logra remi- sión completa de la enfermedad con el uso de esteroides y azatioprina (7).

Al buscar similitudes con el presente caso, se encuentra que ambos se presentaron en mujeres jóvenes; sin embargo, el reportado previamente en la literatura ocurre en una paciente en quien concurren varias enfermedades autoinmunes, a quien se le suministró adalimumab por un tiempo medio de tres meses, a diferencia del presente caso, en el cual la paciente alcanza remisión completa y sostenida de su enfermedad reumatológica con el uso continuo del agente anti-TNF por dos años, antes de evidenciarse elevación en sus transaminasas.

El diagnóstico diferencial de compromiso hepático en artritis reumatoide (AR) incluye: hepatitis inducida por medicamentos, hepatitis viral, hígado graso y hepatitis autoinmune, esta última ocurre en $4.2 \%$ de los pacientes con AR; tiende a ser leve con alteraciones transitorias de las pruebas de función hepática que se correlacionan con la actividad de la enfermedad; pocos pacientes desarrollan disfunción hepática progresiva. Diferenciar la presencia de hepatitis autoinmune en pacientes con AR de compromiso hepático por AR es difícil, ya que los pacientes con hepatitis autoinmune pueden tener manifestaciones extrahepáticas que simulan AR, mientras que la AR se puede asociar a hipergammaglobulinemia y títulos altos de autoanticuerpos. La biopsia hepática permite hacer con mayor claridad el diagnóstico diferencial: la ausencia de necrosis en puente y fragmentaria o la infiltración marcada con plasmocitos favorecen más la presencia de compromiso hepático por AR que el diagnóstico de hepatitis autoinmune (11).

En conclusión, hasta donde se sabe, se está reportando el segundo caso descrito en la literatura de hepatitis autoinmune inducida por adalimumab. Cuando se presenta elevación de transaminasas en pacientes que utilicen este tipo de medicamentos, deben plantarse varios diagnósticos diferenciales, como: hígado graso, uso concomitante de otros hepatotóxicos, como medicamentos y tóxicos, e infecciones oportunistas o crónicas virales no detectadas previamente. En esta lista de probabilidades, debe incluirse la presencia de autoinmunidad hepática.

\section{Fuentes de financiación}

Recursos propios.

\section{Posibles conflictos de interés}

Ninguno de los autores ha recibido ningún incentivo por parte de compañías farmacéuticas u otros patrocinadores.

\section{Referencias}

1. Krawitt EL. Autoimmune Hepatitis. N Engl J Med 2006; 354: 54-66.

2. Czaja AJ, Manns MP. Advances in the Diagnosis, Pathogenesis, and Management of Autoimmune Hepatitis. Gastroenterology 2010; 139: 58 -72.

3. Zimmerman HJ, MD. Drug induced Liver Disease. Clin Liver Dis 2000; 4: 73-96.

4. Eriksson C, Engstrand S, Sundqvist KG. Autoantibody formation in patient with rheumatoid arthritis treated with anti-TNF. Ann Rheum Dis 2005; 64: 403-7. 
5. Desai SB, Furst DE. Problems encountered during anti-tumour necrosis factor therapy. Best Pract Res Clin Rheumatol 2006; 20: 757-90.

6. Navarro VJ, Senior JR. Drug - Related Hepatotoxicity. N Engl J Med 2006; 354: 731-9.

7. Adar T. Adalimumab - induced Autoinmune Hepatitis. Case Report. J Clin Gastroenterol 2010; 44: 20-2.

8. Levälampi T, Korpela M. Etanercept and adalimumab treatment in patients with rheumatoid arthritis and spondyloarthropathies in clinical practice: adverse events and other reasons leading to discontinuation of the treatment. Rheumatol
Int 2008; 28: 261-9.

9. Germano V., Picchianti Diamanti A, Baccano G, Natale E, Onetti Muda A, Priori R, et al. Autoimmune hepatitis associated with infliximab in a patient with psoriatic arthritis. Ann Rheum Dis 2005; 64: 1519-20.

10. Hartmann U, Schmitt S, Reuss-Borst M. Elevated liver enzymes in rheumatoid arthritis: differential diagnostic considerations based on a case report. Z Rheumatol 2008; 67: 440-4.

11. Ebert EC, Hagspiel KD. Gastrointestinal and Hepatic Manifestations of Rheumatoid Arthritis. Dig Dis Sci 2011; 56: 295-302. 